\title{
NEUROADIPOBIOLOGY OF ARRHYTHMOGENIC RIGHT VENTRICULAR DYSPLASIA. AN IMMUNOHISTOCHEMICAL STUDY OF NEUROTROPHINS
}

\author{
Peter I. Ghenev', Martina G. Kitanova', Hristo B. Popov' , Nikolay Evtimov², Stoyan V. Stoev³, \\ Anton B. Tonchev ${ }^{4}$, and George N. Chaldakov ${ }^{4}$ \\ 'Department of General and Clinical Pathology, Forensic Medicine and Deontology, ${ }^{2}$ Urology Clinic, University St Anna \\ Hospital, Varna, Bulgaria, ${ }^{3}$ Department of Forensic Medicine, Medical University, Sofia, Bulgaria, and ${ }^{4}$ Department of \\ Anatomy and Histology, Medical University, Varna, Bulgaria
}

\begin{abstract}
Arrhythmogenic right ventricular dysplasia (ARVD) is an inherited disorder of cardiomyocyte-to-cardiomyocyte adhesion proteins associated with ventricular arrhythmias and sudden cardiac death. It is a characterized by progressive fibrofatty replacement of right ventricular myocardium. The presence of adipose tissue either with or without fibrous tissue, scattered among cardiomyocytes is the histological hallmark of the disease. Being in the myocardium, adipocytes trigger damage to cardiomyocytes, thus causing electrical instability of the right ventricular myocardium, but the molecular pathogenesis of such an electrical instability in ARVD is still unclear. Since (i) adipose tissue replacement of cardiomyocytes is the most essential histological finding in ARVD, (ii) nerve growth factor (NGF) exerts an arrhythmogenic effect related to sudden cardiac death, and (iii) adipose tissue produces NGF and brain-derived neurotrophic factor (BDNF), the aim of the present study is to analyze immunohistochemically ARVD-related adipocytes with special attention to the expression of NGF and related neurotrophins, BDNF and neurotrophin-3 (NT-3) and their respective TrkA, TrkB and TrkC receptors. Eight cases with ARVD were autopsy proven. The present results demonstrate that the intramyocardial adipocytes and cardiomyocytes in ARVD express NGF/TrkA and NT-3/TrkC, suggesting that they may play a substantial part in life-threatening myocardial electrical instability.
\end{abstract}

Adipobiology 2016, 8: 55-58

Key words: arrhythmogenic right ventricular dysplasia, adipose tissue, NGF, BDNF, NT-3, Trk receptors 


\section{Introduction}

Arrhythmogenic right ventricular dysplasia (ARVD), also known as arrhythmogenic right ventricular cardiomyopathy, was first described 40 years ago (1), while analyzing the causes of fatal ventricular arrytmias. Initially, young people were supposed to suffer from the disease, but later on it was established in elderly (2). Arrhythmogenic right ventricular dysplasia a predominantly genetically determined form of cardiomyopathy that is characterized histologically by the replacement of degenerated cardiomyocytes with adipose and fibrous tissue, and leads to arrhythmias, which account for approximately $20 \%$ of sudden cardiac death in the young ( $\leq 35$ years). The estimated prevalence of ARVC in the general population ranges from 1 in 2,000 to 1 in 5,000; men are more frequently affected than women. There have been 12 genes identified which are linked to dysfunctional desmosomes in ARVD. Examples of such defective desmosomal proteins are plakoglobin, desmoplakin, plakophilin-2 and desmoglein-2 (3-9). Recent data reveal that ARVD can be considered as a disease of the intercalated disc, rather than only as a desmosomal disease (10). Whereas in the past, the disease was considered to involve only the right ventricle, more recent clinical studies have established that the left ventricle is also involved, thus the term arrhythmogenic cardiomyopathy has emerged $(9,10)$.

Despite increasing knowledge of the genetic basis of ARVD, we are just beginning to understand the molecular biology of ARVD-associated arrhythmogenic events.

The presence of mature adipocytes either with or without fibrous tissue, scattered among cardiomyocytes is the hallmark of the disease. Since ( $i$ ) adipose tissue replacement of degenerated cardiomyocytes is the most essential histological finding in ARVD, (ii) nerve growth factor (NGF) exerts an arrhythmogenic effect related to sudden cardiac death $(11,12)$, and (iii) adipose tissue produces NGF and brain-derived neurotrophic factor (BDNF) (13), the aim of the present study was to analyze immunohistochemically ARVD-related adipocytes and cardiomyocytes with special attention to the expression of the neutrophins NGF, BDNF and neurotrophin-3 (NT-3) and their respective TrkA, TrkB and TrkC receptors.

\section{Subjects and methods}

The study is based on the autopsy findings in eight cases (five men and three women, age range $26 \div 59$ years), all of them consistent with ARVD. Maximum optimal times after death and proper duration of tissue fixation in $10 \%$ neutral buffered formalin were provided. Gross examination and histological observation of all important organs and tissues was carried out on routinely stained after hematoxylin and eosin 4 micrometer thick tissue sections. In addition, immunohistochemical study of the expression of NGF, BDNF and NT3 and their respective TrkA, TrkB and TrkC receptors was done. Following the standard procedure in regard to antigen retrieval, reagents dilution, incubation time, negative controls, etc, primary antibodies, compatible with FLEX visualization system were loaded in LINK automatic IHC stainer. All specimens were scanned by Aperio Slide scanner and digital images analyzed on a computer.

\section{Results}

ARVD is a rare disorder, but for a short period of time, eight cases were proven by autopsy in a relatively small region in the Northeastern part of Bulgaria. All of them shared similar history - sudden death during minor physical effort without any evidence of prior cardiovascular disease or complaints

The gross findings in all autopsy cases were consistent of signs for rapid occurring death - severe congestion in all viscera, without postmortem blood coagulation, presence of pulmonary and cerebral edema; no other significant lesions were found. Gross changes in the heart in all cases were minimal - slightly dilated cardiac ventricles with spotty endocardial surface and somewhat pale and streaky myocardial cut surface. Major arterial blood vessels showed minor degree of atherosclerotic involvement. Light microscopy findings in the dilated right ventricle myocardium included increased amount of adipose tissue forming massive layers (Fig. 1A) and features of hypertrophy of preserved cardiomyocytes, such as "boxcar" nuclei (Fig. 1B). In all of the cases, no features of inflammation were evident in the heart.

The myocardium of the left ventricle in all cases appeared normal except the presence of "wavy" fibers (data not shown).

Immunohistochemical study revealed positive expression of NGF and TrkA both in intramyocardial adipocytes as small dots at the periphery beneath the cellular membrane and in the cytoplasm of cardiomyocytes also as small dots on both sides of the nucleus (Fig 2. A,B)

Immunohistochemical results of the expression of NT-3 and TrkB are presented on Fig. 3 A,B.

As for the other neurotrophin, BDNF and its receptor TrkB, and also the panneurotrophic receptor $\mathrm{p} 75^{\mathrm{NTR}}$ no positive expression was established (data not shown).

\section{Discussion}

As adipose tissue replacement of cardiomyocytes is the most essential histological finding in all of the autopsy cases, we consider the gross and light microscopy evidence presented above to be consistent with ARVD. All the cases studied here reveal the typical involvement of the heart without any other significant pathological changes to explain sudden death. In spite of the considerable progress in the understanding of the 


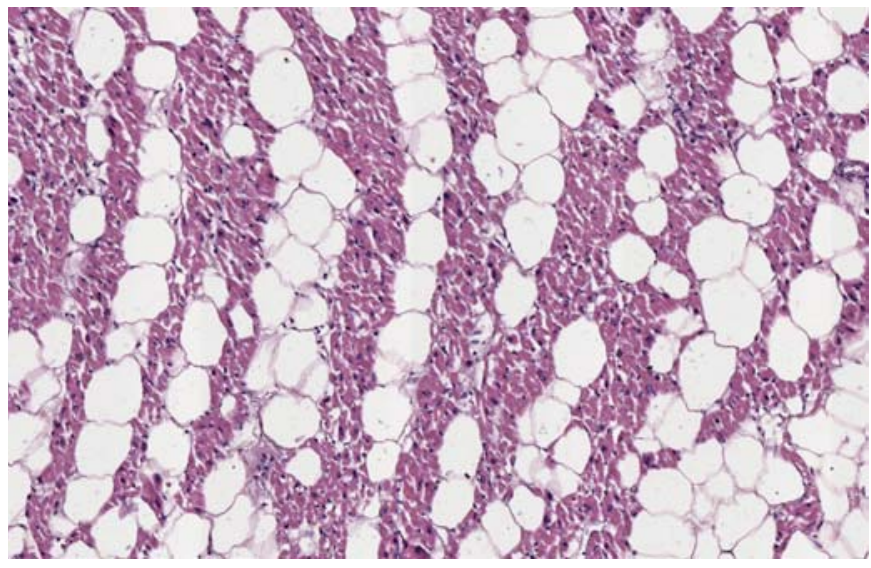

A

Figure 1. Microphotograph of right ventricle myocardium: A, layers of mature adipocytes intermingled with cardiomyocytes; $\mathrm{HE}$, original magnification $\times 100$, and $\mathbf{B}$, hypertrophy of preserved cardiomyocytes with "boxcar" nuclei; HE, original magnification $\times 600$.

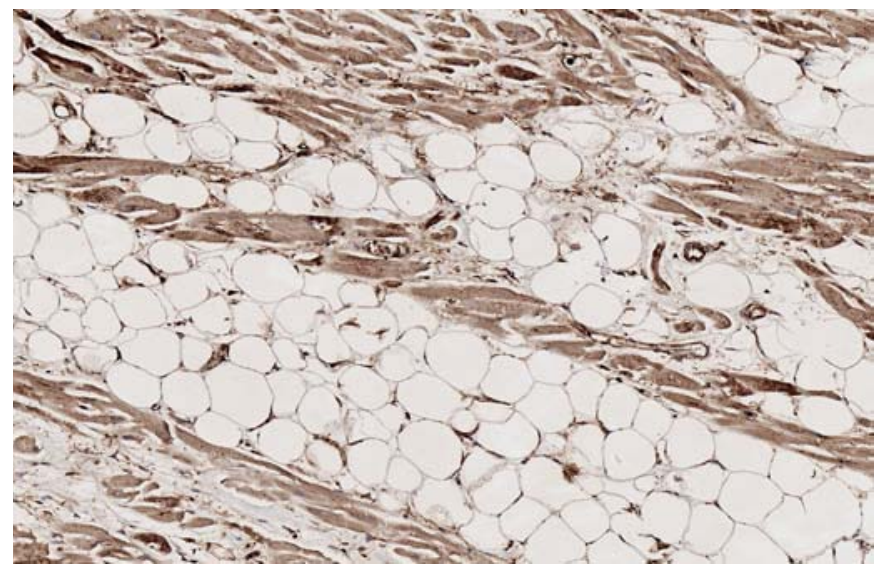

A

Figure 2. Microphotograph of right ventricle myocardium with mature adipocytes; A, positive expression of NGF, and B. of TrkA; original magnification $\times 100$.

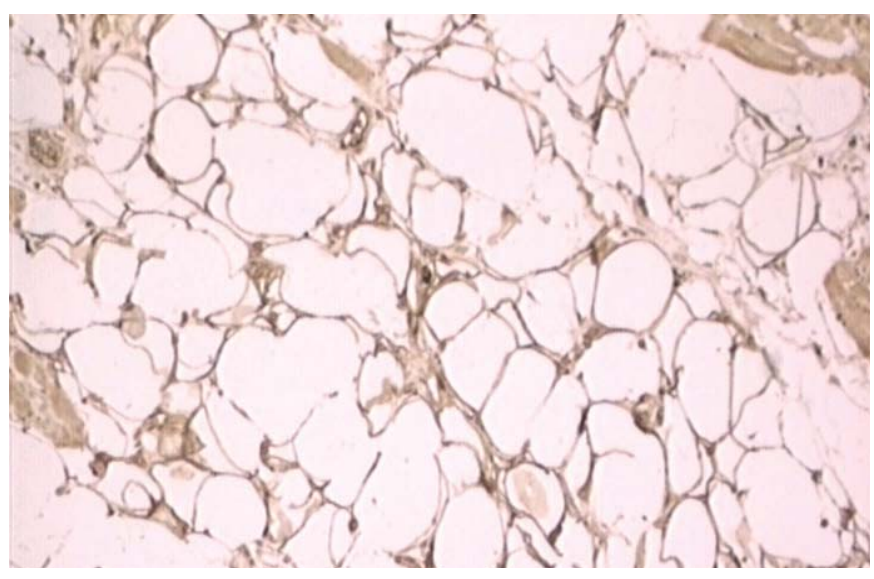

A

Figure 3. Microphotograph of right ventricle; A, positive expression of NT-3 both in cardiomyocytes and adipocytes; original magnification x200, and B, very weak positive expression of TrkC in cardiomyocytes and adipocytes; original magnification x100. 
clinical presentation, the molecular pathogenesis of cardiac electrical instability in ARVD is still unclear.

Whereas NGF and BDNF exert cardioprotective and metabotrophic action (14-17), the expression of NGF/TrkA and NT-3/TrkC in both cardiomyocytes and adipocytes suggests another possible - non-desmosomal - pathogenetic mechanism for myocardial electrical instability in ARVD (also see 11, 12). In that vein, a possibility of TrkA receptor antagonists (18) as potential antiarrhythmic drugs for ARVD may be examined.

Other targets in the pathogenesis of ARVD might be ( $i$ ) NGFmediated connexin phosphorylation of gap (channel) junctions (19), and (ii) neurotrophin- and catecholamine-dependent communication between cardiomyocytes and nerves (20).

\section{Conflict of interest statement}

The authors have no conflicts of interest.

\section{References}

1. Fontaine G, Guiraudon G, Frank R, Vedel J, Grosgogeat Y, Cabrol C, et al. Stimulation studies and epicardial mapping in ventricular tachycardia: study of mechanisms and selection for surgery. In: Kulbertus HE, editor. Reentrant Arrhythmias: Mechanisms and Treatment. Lancaster: MTP Press Limited; 1977; pp 334-350.

2. More D, O’Brien K, Shaw J. Arrhythmogenic right ventricular dysplasia in the elderly. Pacing Clin Electrophysiol. 2002; 25: 1266-1269. DOI: 10.1046/j.1460-9592.2002.01266.x

3. Azaouagh A, Churzidse S, Konorza T, Erbel R. Arrhythmogenic right ventricular cardiomyopathy/dysplasia: a review and update. Clin Res Cardiol. 2011; 100:383-394. DOI: 10.1007/s00392-011-0295-2

4. Tabib A, Loire R, Chalabreysse L, D. Meyronnet, Miras A, Malicier D, et al. Circumstances of death and gross and microscopic observations in a series of 200 cases of sudden death associated with arrhythmogenic right ventricular cardiomyopathy and/or dysplasia. Circulation 2003; 108: 30003005. DOI: 10.1161/01.CIR.0000108396.65446.21

5. Matolweni LO, Bardien S, Rebello G, Oppon E, Munclinger M, Ramesar R, et al. Arrhythmogenic right ventricular cardiomyopathy type 6 (ARVC6): support for the locus assignment, narrowing of the critical region and mutation screening of three candidate genes. BMC Med Genet 2006; 7: 29. DOI: 10.1186/1471-2350-7-29

6. Thiene G, Corrado D, Basso C. Arrhythmogenic right ventricular cardiomyopathy/dysplasia. Orphanet $J$ Rare Dis 2007; 2: 45-61. DOI: 10.1186/1750-1172-2-45.

7. Awad MM, Calkins H, Judge DP. Mechanisms of disease: molecular genetics of arrhythmogenic right ventricular dysplasia/cardiomyopathy. Nat Clin Pract Cardiovasc Med 2008;
5: 258-267. DOI: $10.1038 /$ ncpcardio1182

8. Yang Z, Bowles NE, Scherer SE, Taylor MD, Kearney DL, $\mathrm{Ge} S$, et al. Desmosomal dysfunction due to mutations in desmoplakin causes arrhythmogenic right ventricular dysplasia/cardiomyopathy. Circ Res 2006; 99: 646-655. DOI: 10.1161/01.RES.0000241482.19382.c6

9. Fontaine G, Chen H-s V. Arrhythmogenic right ventricular dysplasia back in force. Am J Cardiol 2014; 113: 1735-1739. DOI: 10.1016/j.amjcard.2014.03.001

10. Calore M, Lorenzon A, De Bortoli M, Poloni G, Rampazzo A. Arrhythmogenic cardiomyopathy: a disease of intercalated discs. Cell Tissue Res 2015; 360:491-500. DOI: 10.1007/ s00441-014-2015-5

11. Cao JM, Chen LS, KenKnight BH, Ohara T, Lee MH, Tsai J, et al. Nerve sprouting and sudden cardiac death. Circ Res. 2000; 86: 816-821. DOI:10.1161/01.RES.86.7.816

12. Bloom HL. Beyond beta-blockade: Nerve growth factor and arrhythmia. Heart Rhythm 2007; 4: S437-S448. DOI: 10.1016/j.hrthm.2007.06.017

13. Sornelli F, Fiore M, Chaldakov GN, Aloe L. Adipose tissuederived nerve growth factor and brain-derived neurotrophic factor: results from experimental stress and diabetes. Gen Physiol Biophys 2009;28:179-183.

14. Levi-Montalcini R. The nerve growth factor 35 years later. Science 1987; 237: 1154-1162. DOI: 10.1126/science.3306916

15. Aloe L, Chaldakov GN. The multiple life of nerve growth factor: tribute to Rita Levi-Montalcini (1909-2012). Balkan Med J 2013; 30: 4-7. DOI: 10.5152/balkanmedj.2013.003]

16. Abe T, Morgan DA, Gutterman DD. Protective role of nerve growth factor against postischemic dysfunction of sympathetic coronary innervation. Circulation 1997; 95: 213-220. DOI: 10.1161/01.CIR.95.1.213

17. Chaldakov GN, Fiore M, Stankulov IS, Manni L, Hristova MG, Antonelli A, et al. Neurotrophin presence in human coronary atherosclerosis and metabolic syndrome: a role for NGF and BDNF in cardiovascular disease? Prog Brain Res 2004; 146: 279-289. DOI: 10.1016/S0079- 6123(03)46018-4

18. Yanev S, Fiore M, Hinev A, Ghenev PI, Hristova MG, Panayotov $\mathrm{P}$, et al. From antitubulins to trackins. Biomed Rev 2016; 27: 59-67.

19. Cushing P, Bhalla R, Johnson AM, Rushlow WJ, Meakin SO, Belliveau DJ. Nerve growth factor increases connexin43 phosphorylation and gap junctional intercellular communication. J Neurosci Res 2005; 82:788-801. DOI: 10.1002/jnr.20689

20. Franzoso M, Zaglia T, Mongillo M. Putting together the clues of the everlasting neuro-cardiac liaison. Biochim Biophys Acta. 2016; 1863(7 Pt B):1904-1915. DOI: 10.1016/j. bbamcr.2016.01.009. 